\section{Cahiers de Narratologie}

Analyse et théorie narratives

22 | 2012

Voix off et narration cinématographique. Second volet

\title{
Voix-off et silences chez Isabel Coixet
}

\author{
Silvina Bénévent González
}

\section{(2) OpenEdition}

Journals

Édition électronique

URL : http://journals.openedition.org/narratologie/6548

DOI : $10.4000 /$ narratologie. 6548

ISSN : $1765-307 X$

Éditeur

LIRCES

\section{Référence électronique}

Silvina Bénévent González, « Voix-off et silences chez Isabel Coixet », Cahiers de Narratologie [En ligne], 22 | 2012, mis en ligne le 20 juillet 2012, consulté le 30 avril 2019. URL : http://

journals.openedition.org/narratologie/6548; DOI : 10.4000/narratologie.6548

Ce document a été généré automatiquement le 30 avril 2019

Article L.111-1 du Code de la propriété intellectuelle. 


\title{
Voix-off et silences chez Isabel Coixet
}

\author{
Silvina Bénévent González
}

1 Tout spectateur et tout critique manipule cette expression de la voix-off comme si elle allait de soi et comme si elle n'existait que dans une relation complémentaire à l'image. Cependant, Michel Chion a montré combien les fonctions de la voix-off s'avèrent être multiples. De facto, le recours presque systématique à ce procédé dans l'œuvre d'Isabel Coixet, cinéaste espagnole d'envergure, nous a incitée à nous interroger sur la fonction, les effets de la voix-off et les rapports qu'elle entretient avec la narration proposée par la réalisatrice.

2 Certes de prime abord, cette utilisation récurrente laisse présupposer une unité de sens, mais la gageure est aussi de démontrer une fonction de la voix-off propre à la réalisatrice. Pour ce faire, nous avons concentré notre attention sur le corpus suivant : A los que aman (1998), La vida secreta de las palabras (2005) et Mapa de los sonidos de Tokio (2009) sans pour autant nous interdire quelques incursions dans Cosas que nunca te dije (1996) et Mi vida sin mí (2003).

3 A priori, Isabel Coixet choisit des récits dissemblables et un cadre spatio-temporel différencié. En effet, le récit du premier film se situe dans l'Espagne du XVIII ème siècle, le second met en scène des êtres isolés sur une plateforme pétrolière perdue dans la mer $\mathrm{du}$ Nord, tandis que le dernier nous transporte dans le Japon contemporain. L'apparente diversité des sujets pose de suite le problème de ce que l'on nomme généralement la bande sonore.

4 Il s'agit alors dans un premier temps d'analyser quelles sont les modalités d'apparition de la voix-off et son rapport au narrateur. Mais à vrai dire, l'inclusion de la voix-off dès la première séquence marque déjà un acte de parole, dans une certaine mesure, pré-existant à l'image. Et c'est en cela que la voix-off est un lien entre le subjectif et l'objectif, entre le corps et le code, ce qu'il conviendra d'analyser dans une deuxième partie. Enfin, une troisième partie établira comment la voix-off révèle après coup la douleur de la situation traumatique, au sein d'une structure paradoxale d'une voix disparue rendue présente. 


\section{La voix-off et ses modalités}

5 quel magma sonore, la présence d'une voix humaine hiérarchise la perception autour $\mathrm{d}^{\prime} \mathrm{elle}^{1} »$. Le vococentrisme de l'écoute humaine traduit en effet tout l'enjeu de la voix-off, cette voix dont la source, hors-champ, est non visualisée.

Dans les trois films d'Isabel Coixet de notre corpus, la voix-off possède un signe distinctif tant au niveau du sexe, de l'âge que de la tessiture. Or elles sont toutes extrêmement humaines et engageantes. C'est que la voix-off pose, de prime abord, des repères pour le spectateur et contribue à la mise en place du récit. Quoiqu'elle entretienne un rapport anormal, car non naturel, avec celui qui l'écoute, elle est pourtant perçue, acceptée comme extrêmement crédible et ce par sa nature même et sa fonction particulière.

Examinons à présent le premier de ces films, A los que aman², dans lequel le schéma de la voix-off est, somme toute, classique : elle retrace le passé de celui qui parle et réactive les sensations qu'il a perçues à l'époque. Selon une classification que nous reprenons de JeanLouis Alibert ${ }^{3}$, le narrateur, présent dans la diégèse, construit donc un récit autodiégétique et assume pleinement la charge d'accompagner les images.

Néanmoins, par un jeu d'apparitions, de disparitions, il cumule plusieurs fonctions de l'acousmêtre telles que les a définies Michel Chion. L'acousmêtre, «c'est la voix cachée, la voix sans visage et ses pouvoirs magiques ${ }^{4}$ ». Le narrateur de A los que aman est ainsi d'abord acousmêtre intégral, «celui qui n'est pas encore vu, mais qui reste susceptible d'apparaître à tout moment dans le champ ${ }^{5}$ ». En effet, la voix-off qui entame le récit partage les réflexions et les émotions d'un acousmêtre absent, telle une voix de la conscience, une voix intérieure. Dans le même temps, à l'image, le regard du spectateur suit le mouvement des nuages dans le ciel en plan subjectif. La corrélation de l'image et du commentaire invite en somme à l'introspection et établit d'ores et déjà la perspective subjective induite par la narration nostalgique.

Ensuite, l'attente du spectateur - qui ressent la nécessité d'une forme animée à coupler à la voix-off - est immédiatement satisfaite : la source de la voix-off est identifiée sous les traits d'un vieil homme faisant la leçon à des enfants sous un arbre. En voix-off, il se déclare témoin et acteur du drame passionnel qui va se dérouler sous nos yeux: «J'ai passé ma vie à aimer une femme qui aimait un homme qui, lui, ne l'aimait pas. Il aimait une autre femme et je n'ai jamais su si celle-ci l'aimait en retour ${ }^{6} »$. En réalité, le spectateur élabore seul cette identification de la voix-off et du vieil instituteur, par simple similitude de la voix-off et de la voix-in.

Une fois cette désacousmatisation effective; l'acousmêtre déjà-vu, «temporairement sorti du champ", laisse place à la mise en images de son discours. Dès les premières minutes, l'image des nuages dans le ciel, puis d'une demi grenade et enfin la course des enfants dans un pré le ramènent au souvenir de sa rencontre avec Matilde alors qu'ils étaient encore enfants. Figure essentielle, la voix-off se place en somme en prolepse du récit filmique qu'elle va composer tout en s'articulant comme anamnèse de la narration.

11 Le narrateur navigue de la sorte entre différents types de voix narratives pour assurer la sensation d'intimité avec son interlocuteur, et ce d'emblée. Les paroles du narrateur accompagnent dès lors le jeune homme qu'il a été, puis, lorsqu'enfant, il avait connu son premier émoi amoureux, pour finalement revenir au vieil homme qu'il est devenu et qui 
se retourne sur sa vie. La pierre angulaire du récit du personnage-narrateur est l'amour qu'il a éprouvé pour Matilde, un amour déçu puisqu'elle s'éprend de Léon, un jeune Français, et l'épouse. La rencontre entre les futurs époux est le fruit d'une série de confusions sentimentales dont le narrateur est le témoin passif voire le soutien accidentel 7. Les confusions persistent et mènent le jeune Français à entretenir une folle passion avec Valeria, son maître d'escrime. L'inévitable découverte de l'adultère sera la cause du dépérissement de Matilde que le narrateur est impuissant à sauver. Moteur involontaire d'un destin contraire à ses vœux, le narrateur se sacrifie littéralement et reste le confident de Matilde jusqu'à sa mort.

Pourtant, la réalisatrice prend soin d'établir l'introspection- rétrospection du narrateur comme nécessaire à l'économie narrative. Le récit est effectivement déclenché par la curiosité d'un jeune homme; il est le fils d'Armancia, la sœur cadette de Matilde. Le destin se montre ici malicieusement redondant puisque sa femme souffre du même mal que Matilde. Ébranlé par la situation et encouragé par les questions du jeune homme, le personnage-narrateur choisit alors l'action (narrative) et se lance à ce moment-là dans un récit salvateur et rédempteur pour lui-même. Dans la continuité retrouvée d'un récit du passé, l'inclusion essentielle de la voix-off en surimpression sur l'image se présentera dès lors à nous, comme une trace mémorielle intacte et unique, car personne ne vient confirmer ni invalider ses affirmations. Le narrateur s'installe ainsi dans sa voix et oriente pour nous événements et discours d'une voix forte et puissante tout en instaurant l'omnipotence de la parole à l'unisson de la leçon qu'il donne à ses jeunes élèves : « On dit qu'à travers les mots, la douleur devient plus tangible. Pour ma part, j'ai toujours cru que la souffrance qui ne trouve pas de mot pour être exprimée est la plus cruelle, la plus profonde et la plus injuste des souffrances ${ }^{8} "$.

Par un jeu continuel de mise-en-corps et d'acousmatisation de la voix narratrice, le récit acquiert une cohérence naturelle et installe solidement le principe de vraisemblance face à une parole mensongère (celle de Léon et du fils d'Armancia, époux infidèles), une parole déraisonnée (le discours du frère du narrateur est désorganisé) et une parole éteinte (Matilde n'est plus). La voix-off dispensatrice de l'image fait ainsi le lien entre toutes les voix.

14 Nous distinguerons un deuxième cas de figure dans Mapa de los sonidos de Tokio ${ }^{9}$, dans lequel un homme témoigne de la vie d'une disparue, Ryu, et maintient de la sorte son souvenir. Tel un psychopompe, il s'entête à redonner vie à celle qui a disparu, au nom de leur amitié. Comme dans le film antérieur, l'implication de soi dans le récit est à nouveau un pré-requis, à une différence près. Le personnage-narrateur, anonyme, évolue à l'image dans le silence. C'est un acte de parole qui n'est jamais visualisé puisque le narrateur homodiégétique ne remue pas les lèvres à l'écran.

En ce qui concerne La vida secreta de las palabras, une voix de petite fille, en assumant, elle aussi, pleinement le préambule, laisse penser au spectateur qu'il va assister à un long flash-back. Toutefois, en repoussant indéfiniment la jonction de la voix et de la figure animée, le récit entretient l'indéfinition de son identité. Elle est une source mystérieuse et absente, jusqu'à être une présence en creux. Elle fixe ainsi sa fonction de narrateurcommentateur hétérodiégétique comme absent de la diégèse filmique ou issu peut-être d'un autre univers diégétique.

16 Toutes ces voix narratives se donnent donc pour fonction d'être la cause du récit autant que sa source. On peut remarquer dans un deuxième temps qu'elles s'affirment toutes dans un «je » qui parle d'un « elle », disparue, jusqu'à éluder, semble-t-il, un «nous » 
impossible. À ce titre, le personnage énonciateur s'exerce entre le son et l'image au sein du discours qu'il produit et instaure l'objet de ce discours (la femme disparue) en se revendiquant sujet anonyme et unique du discours.

À l'exemple de Mapa de los sonidos de Tokio, le spectateur est projeté, par la voix-off, dans l'univers diégétique. Il se sent accaparé par cette vision unidirectionnelle du drame et il a, en même temps, l'impression d'une histoire en train de se faire. Le procédé vise en fin de compte à emporter son adhésion, à le capturer dans son univers par le seul biais de la voix-off. Elle met en place ce que Gérard Genette nomme la focalisation, ce mode narratif qui est "une restriction de "champ" c'est-à-dire en fait une sélection de l'information narrative ${ }^{10} »$. Dans A los que aman et Mapa de los sonidos de Tokio, elle unit en l'occurrence deux dimensions contradictoires, celle de voir et de se voir. Le «je » se double quand le narrateur se met en scène et le narrateur, sorti du champ, laisse place à un reflet de luimême. Non seulement il revoit les événements qu'il raconte, mais il se revoit tel qu'il a pu être et tel qu'il s'imagine avoir été. Par ce jeu, la figure du narrateur est démultipliée. Dans A los que aman, la voix d'aujourd'hui se juxtapose aussi à la voix d'antan dans une continuelle auto-affirmation de soi et plusieurs temporalités se superposent alors comme autant de niveaux de narration. De fait, la voix-off assujettit littéralement l'image du locuteur à lui-même, un locuteur engendré par son seul discours, au-delà de la simple utilisation de la première personne du singulier.

Bien plus, la voix narrative partage avec le spectateur ses doutes et ses hésitations et établit humblement le peu de fidélité qu'il faut prêter à sa mémoire. Le narrateur de A los que aman avoue "C'est ce dont je crois me souvenir", et celui de Mapa de los sonidos de Tokio surénchérit : "J'en suis presque sûr ${ }^{11}$ ». Faire naître le doute équivaut en d'autres termes à enraciner le récit dans le terreau de la mémoire et de l'imagination, ce qui contribue à « l'effet de réel », selon l'expression consacrée de Roland Barthes. De la même façon, l'interpellation de l'enfant dans La vida secreta de las palabras, «Je vous l'ai déjà dit, non $^{12}$ ?» s'avère être une prise à témoin, un questionnement du spectateur, une métalepse qui l'accompagne et parle exclusivement pour lui.

Toutefois, parler implique nécessairement un interlocuteur. Dans Mapa de los sonidos de Tokio, le spectateur est comme invité à surprendre le locuteur de dos, puis de profil, mais en aucun cas de face. C'est précisément parce que ce corps n'est pas entraîné vers l'acte de narrer qu'il se présente plutôt d'emblée dans la contemplation, dans la tranquillité d'un cimetière, dans une fixité étonnante du présent. 
Mapa de los sonidos de Tokio (photo de presse)

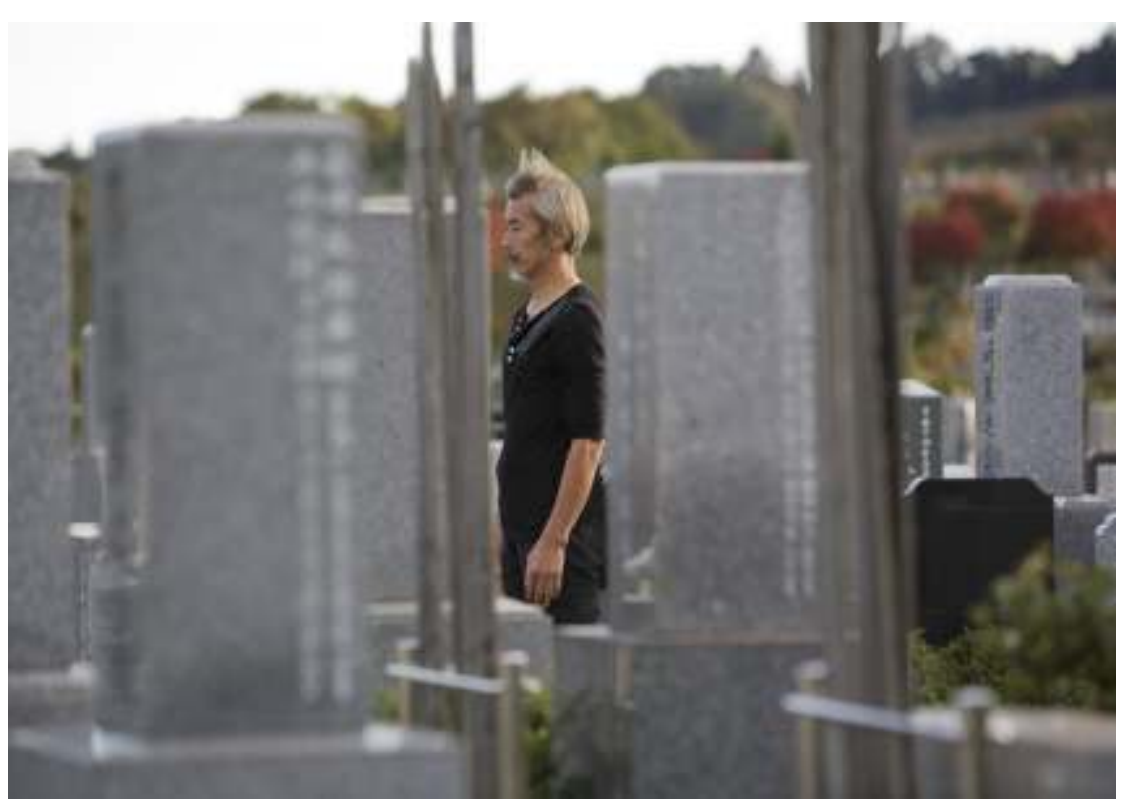

Figure 1

(C) 2009. MEDIAPRODUCCIÓN S.L.U. I VERSÁTIL CINEMA S.L. Fotógrafo : David Coll Blanco

20 À charge donc pour le spectateur d'associer le corps visible d'un homme qui ne parle pas et la voix-off parfaitement audible. Précisons d'emblée que cette dernière est marquée par une légère réverbération acoustique. Si cette technique tient d'une recherche esthétique particulière, elle entraîne, à notre sens, davantage le récit vers la confession. Le même effet technique se répète dans A los que aman, Cosas que nunca te dije, Mi vida sin mí et La vida secreta de las palabras. Mais, il faut également souligner que la voix-off de Mapa de los sonidos de Tokio est accompagnée par le chant des oiseaux ce qui n'est pas étonnant en soi puisque le narrateur se trouve dans le cimetière où Ryu est enterrée. Toutefois, il y a une inadéquation entre les deux sources de son sans que le spectateur n'en soit véritablement surpris : l'un est intérieur, l'autre extérieur.

21 Pour en revenir au montage de cette séquence, on peut facilement déterminer que la prise de parole par le personnage-narrateur débute depuis un temps présent et il avoue regretter son amie Ryu. À ce présent narratif, succède alors un flash-back qui concentre toute l'attention sur Ryu. La caméra déambule dans les allées et les étals de ce qui pourrait être l'immense marché aux poissons de Tsukiji ${ }^{13}$ où travaillait de nuit Ryu. On la découvre en habit de travail vaquer à ses occupations parmi d'autres employés auxquels elle n'adresse jamais la parole. La disparition, durant un temps, de la voix-off fait correspondre le son à l'image de son origine vue : le jet d'eau d'un tuyau d'arrosoir, la scie électrique, etc. Ce synchronisme logique est à nouveau interrompu par le récit du narrateur («Quand je fis sa connaissance, elle s'appelait Ryu [...] nous nous sommes connus un dimanche après-midi au Musée du Ramen $\left.{ }^{14} »\right)$. S'immisce alors le premier contact visuel entre le narrateur et Ryu. Sujet et objet se font presque face, se regardent avec dissimulation et engloutissent bruyamment une soupe : l'échange est visuel. 
Mapa de los sonidos de Tokio (photo de presse)

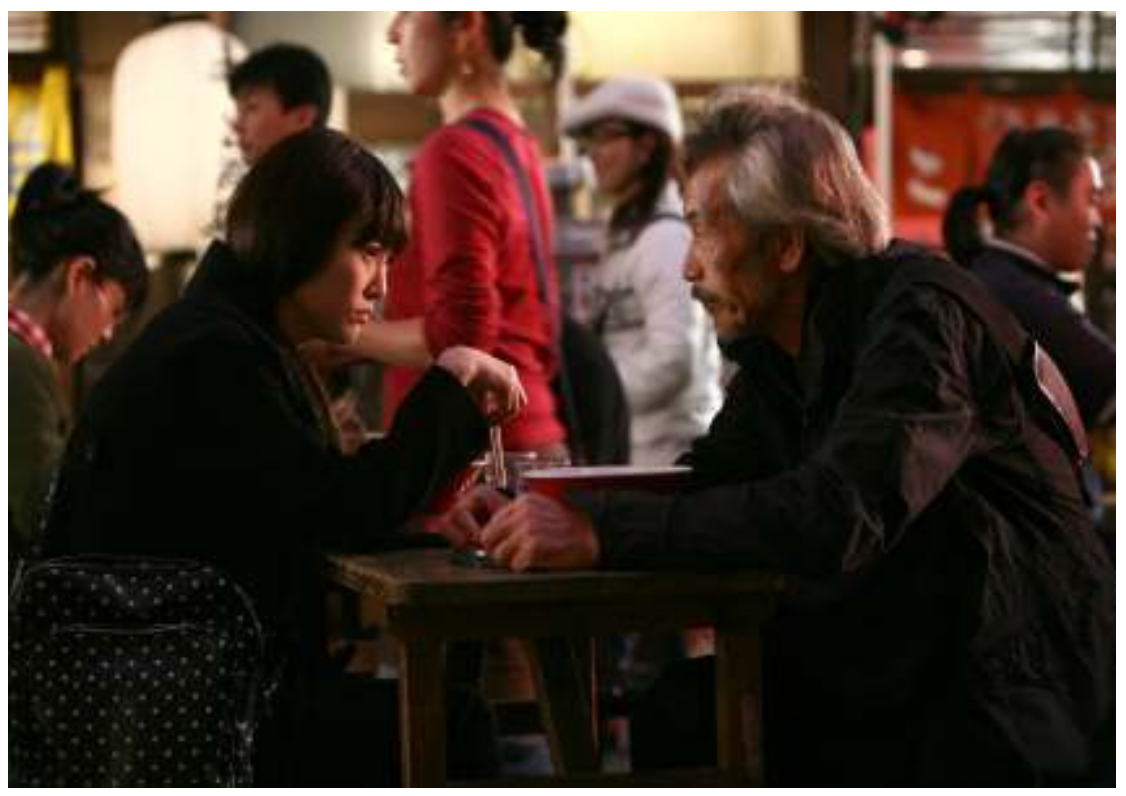

Figure 2

(c) 2009. MEDIAPRODUCCIÓN S.L.U. I VERSÁTIL CINEMA S.L. Fotógrafo : David Coll Blanco

L'échange verbal se superpose ensuite quand la voix-off retranscrit leur première rencontre. L'effet de réverbération cesse alors. Au contraire, les voix de Ryu et du narrateur se rapprochent et se répondent: «Tu me laisses enregistrer le bruit que tu fais? En réalité, c'est mon travail. C'est comme ça que je gagne ma vie. - C'est comme ça que tu gagnes ta vie ${ }^{15}$ ? ». Il l'écoute et s'écoute lui parler, car le lien se tisse par les voix et les bruits corporels, sans que les deux interlocuteurs ne se parlent à l'écran.

Dans cet échange, il y a ainsi discordance de la synchronisation par la transgression d'une convention implicite (l'adéquation bande-son / bande-image). Et pourtant, chacun de ces espaces est relié au souvenir de la rencontre avec Ryu, l'un d'un point de vue visuel, l'autre d'un point de vue auditif. Au delà du fait qu'il donne davantage de relief à l'image, ce collage installe la relation qui sera la leur: la réserve de Ryu est annoncée et l'ingérence du personnage-narrateur dans sa vie. Les personnages parlent au spectateur tout près sur un ton de confidence. En d'autres termes, l'effet primordial de ce procédé est aussi de revendiquer une distance moindre avec lui. Si « la voix off sait tout de l'avant et de l'après de l'action ${ }^{16} »$, - ce en quoi elle semble surplomber l'image, remplir un espace vide dans un mouvement dynamique - elle dérange en même temps la conception du monde diégétique. Néanmoins, ces changements intempestifs sont acceptés tant que la véracité a été établie précédemment.

À l'évidence, chez Isabel Coixet, la voix-off ne se constitue et ne reconstitue le récit que par opposition au silence. Dans Mapa de los sonidos de Tokio, le narrateur, en faisant revivre Ryu par sa parole, sollicite l'émotion à travers la visualisation, l'évocation d'une morte : « Il n'y a pas un jour où je ne me souviens pas de la respiration de Ryu, pas un jour où je ne regrette son silence ». Par son témoignage, il pallie le silence du narratif, la difficulté de la confidence de la jeune femme, car il insiste : « Nous mangions ensemble toujours en silence $^{17}$ ». Ainsi, la voix-off, parfois extérieure à l'action, investit le mouvement tout 
entier en donnant du sens à la parole, celle du narrateur et celle des autres : elle est véritablement une voix fondatrice.

Le narrateur est, du reste, un ingénieur du son qui s'obstine à capter les sons de la mégalopolis de Tokyo, fonction contenue dans le titre même. Il est dès lors doublement engagé dans le mouvement de la trace, transcripteur du mouvement d'autrui. Il cumule en fait tous les pouvoirs que Michel Chion attribuait, fort à propos, à l'acousmêtre : pouvoir d'ubiquité (son regard suit Ryu), de panoptisme (il sait tout des rencontres amoureuses de Ryu), d'omniscience (il devine les sentiments de Ryu) et de toutepuissance (il guide l'action).

En définitive, dans les trois films, la voix-off renvoie à un personnage au centre d'un mystère mais non au centre de l'image. La voix-off est le medium d'un jeu de présence/ absence du corps des morts, des mortes en l'occurrence, à travers la parole vivante. Mais, en fin de compte, elle établit tout aussi clairement des doubles niveaux temporels et spatiaux, dans le seul but d'intensifier le sentiment d'intimité avec le spectateur.

Or, cela suggère également le lien qui s'établit par la voix-off entre le subjectif et l'objectif, entre le corps et le code. Et c'est ce que nous nous proposons d'étudier plus précisément dans La vida secreta de las palabras et Mapa de los sonidos de Tokio.

\section{La voix-off et les sens}

L'élaboration de la première séquence de La vida secreta de las palabras mérite toute notre attention car elle suscite davantage de questions qu'elle ne les clarifie.

Rappelons tout d'abord qu'à chaque fois qu'il y a intervention de la voix-off, le spectateur projette mentalement la voix entendue sur l'espace vu et peut difficilement s'empêcher d'appliquer le principe de cause à effet à la projection mentale.

Or, la figure humaine qui accompagne les paroles prononcées par une voix d'enfant dans La vida secreta de las palabras (" Je vous l'ai déjà dit, non? Il y a si peu de choses. Le silence. Et les mots») est un homme. La projection mentale est pervertie, la stratégie du spectateur échoue a priori mais l'installe a fortiori dans l'espoir d'identifier cette voix. Il y perçoit là la clef de voûte du film. Est-ce la voix d'une protagoniste lorsqu'elle était enfant? Est-ce la voix de l'âme, une voix intérieure, la voix de la conscience? La réalisatrice affirme seulement en quatrième de couverture du scénario : «Quelqu'un a dit que celui qui possède une vie intérieure mène à partir de cet instant une double vie ${ }^{18}$ ». Rien de tangible donc.

31 Disons pourtant que la voix, avant même de s'installer dans un corps, est un espace sensoriel, un pré-lieu de sensations kinesthésiques ${ }^{19}$ " sans le secours de l'image malgré la tentation de ramener le son à sa source tangible, pour "l'objectiviser"20" ".

Les premières minutes de La vida secreta de las palabras illustre ce dernier aspect d'une manière remarquable. Avant d'être une histoire d'amour entre Hanna, l'infirmière, et Josef, l'homme blessé, c'est un film sur les mots et le silence comme le dévoile déjà le titre.

33 Au générique ${ }^{21}$ succède l'image d'une mer encore indéterminée, de nuit, agitée par la houle. Puis le montage cut fait alterner trois plans de la plate-forme pétrolière (qui se rapprochent et s'éloignent de la structure métallique), et des plans d'écran noir. Ces fondus au noir, loin de marquer une fin de séquence, renforcent l'impression d'un regard 
qui se serait posé sur la scène et rappellent des paupières qui se ferment. Qui plus est, le spectateur visualise la scène depuis un bateau que la plate-forme surplombe et que la houle ferait tanguer. Difficile de déterminer qui visualise la scène.

Quant à la bande-son, en toute logique, la toute première image est accompagnée par le bruit assourdissant de la houle. Tel est l'espace d'émission d'une voix anonyme qui semble émerger de nulle part, comme détachée d'un corps inaccessible. Dans l'espace acoustique d'apparition de la voix narrative, la lisibilité se donne donc dans la disparition $\mathrm{du}$ fond sonore (le bruit de la mer) derrière la musique ${ }^{22}$ et derrière la voix, qui s'envisage d'abord comme un murmure, une trace de voix, un temps asexuée. Pourtant, le scénario de La vida secreta de las palabras indique "C'est une voix d'une grande pureté qui parle depuis un espace au-delà du "ici et maintenant" que les images laissent voir ${ }^{23}$ ». Le glissement depuis un espace visible (celui de la plate-forme) vers un monde intérieur s'énonce via la voix-off - là encore entendue en réverbération. Par un effet de nonconcordance de l'image et de la voix hors-champ, le spectateur concentre ainsi spontanément son attention sur le texte énoncé que l'on semble lui susurrer à l'oreille ${ }^{24}$.

Comme dans chacun de ses films, Isabel Coixet suggère au spectateur qu'il capte là un fragment de récit, quelque peu tronqué, ce qui a pour effet d'accentuer l'isolement de la voix-off émise depuis un espace autre que celui que montrent les images. La surprise persiste quand la voix narratrice accumule des substantifs, des mots sans connexion et des termes parfois antithétiques comme un démembrement du langage. Ou s'agit-il d'un langage secret?

Soulignons que la voix-off a banni les pauses signalées dans le scénario par un point, elle reprend à peine son souffle d'où sa rapidité d'élocution.

D'emblée, cette voix narratrice s'affirme dans une reconquête des mots face au silence, au non-dit ou au néant. Et ces quelques remarques sont essentielles dans l'œuvre d'Isabel Coixet qui ménage toutefois des associations complexes entre image et son. En effet, dès les premières images, elle joue sur cette idée d'un hors-lieu et d'un hors-temps à la fois provisoires et infinis depuis lequel une voix d'enfant vibre et chercherait à se faire entendre "Maintenant. Tout de suite. Il y a un moment ». Il s'agit d'une voix qui n'établit pas un récit rétrospectif mais énonce la simultanéité des temps jusqu'à atteindre l'intemporalité : «Cent minutes. Mille ans ».

Plus remarquable, on observera que l'élément aquatique ne manque pas d'être ce lien déficient entre la voix et l'image : « Des millions et des millions de tonnes d'eau ».

Entre le visible et l'invisible, la plate-forme fait office de refuge, et renvoie au vide et au plein en évoquant la partie immergée : « Il y a si peu de choses tout au fond ${ }^{25}$ ». 
La plate-forme pétrolière de La vida secreta de las palabras

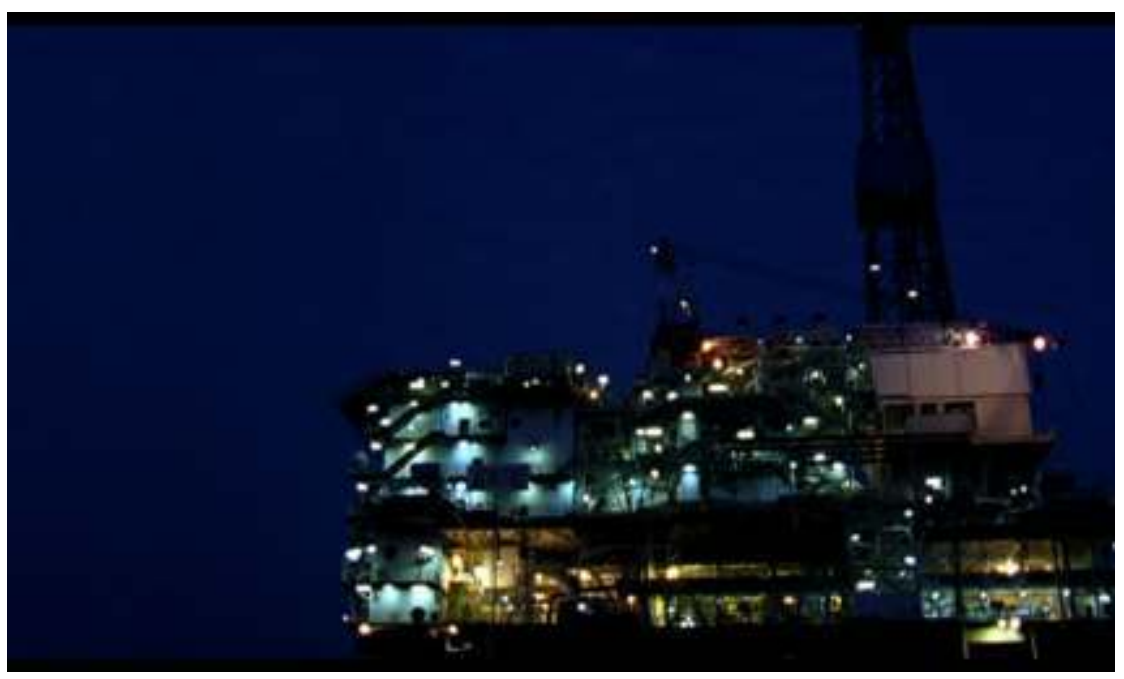

Figure 3

(c) EL DESEO, D.A., S.L.U

40 À la suite de Michel Chion, il nous faut évoquer le lien du sonore avec la scène primitive : l'enfant, dans le monde intra-utérin, capte, perçoit avec netteté la voix de sa mère. Ici, le lien auditif est inversé. Les paroles de l'enfant, « Il y a si peu de choses tout au fond. Des millions et des millions de tonnes d'eau. Des pierres et du gaz. De l'affection. Du sang. Cent minutes. Mille ans ", renvoient à l'intemporalité du monde intra-utérin. Mais l'obscurité et l'humidité peuvent elles aussi, symboliser ce lieu idéal. Réellement désincarnée voire non encore incarnée, la voix-off invite à l'écoute de l'univers intérieur peut-être anténatal.

41 Dans la deuxième partie de la séquence, la voix-off poursuit sa déclamation et l'on assiste à l'apparition d'un incendie sur la plate-forme, tandis que la voix scande « Des cendres. De la lumière ». Ainsi l'élément antithétique de l'eau surgit brutalement et les flammes, la fumée apparaissent à leur tour, par intermittence avec les fondus au noir jusqu'au visage de Josef filmé en caméra à l'épaule, un visage marqué par une grimace de douleur. Du même coup, le fond sonore se transforme et passe du silence à un brouhaha diffus de cris, de crépitements, de pas précipités. Dans la confusion et l'éloignement, le fond sonore de l'accident est le son d'une illusion, une hallucination en résonnance avec une image au ralenti, puisqu'on réserve l'exclusivité de la limpidité à la voix-off. Le feu qui dévore une partie de la plate-forme est en effet le déclencheur du drame (la mort d'un homme et les blessures de Josef) qui est à l'origine de la venue d'Hanna, infirmière de formation, sur la plate-forme. En somme, la voix-off investit l'espace tout entier au-delà d'un arrière-fond sonore guère tenu en compte, et revendique là sa relation au monde et à son espace : « Il y a bien peu de choses. Le silence. Et les mots ", naissance et pré-naissance liées sans commencement ni fin.

Un rapport similaire s'établit dans Mapa de los sonidos de Tokio. Le film débute (avant le générique ${ }^{26}$ ) par le gros plan d'une jeune fille blonde dénudée et allongée sur une table. Par un long travelling en plongée totale depuis le plafond, la caméra balaie le lieu dans un but descriptif, mais pas seulement, il s'agit de s'approprier l'espace de ce restaurant de Tokyo prisé par les Européens mais détesté par Monsieur Nagara, homme d'affaires japonais. Le travelling nous conduit jusqu'à lui, en conversation avec son collaborateur 
Ishida. À ce moment-là, les deux hommes se parlent à voix basse sans se regarder pourtant, à peine gênés par le bruit lointain des voix caverneuses des Européens. Après un coup de téléphone mystérieux, Ishida revient s'asseoir - cette fois derrière Nagara -, et lui murmure quelque chose à l'oreille. La caméra crée ainsi un contact visuel entre les deux hommes tout en se désolidarisant de ce qu'ils se disent car le spectateur en est exclu. Soudain, les cris de Monsieur Nagara «Pourquoi l'a-t-elle fait ${ }^{27}$ ? " sont entendus en forte réverbération et son accès de désespoir est filmé au ralenti, ce qui n'est plus le cas des autres convives, étrangers au drame qui se déclare. Les qualités acoustiques propres à chacun, établies en début de séquence par une post-synchronisation évidente, sont bousculées, et le décalage devient encore plus visible par l'utilisation improbable et déformante du ralenti qui exprime la souffrance d'un père meurtri. L'ultime invitation à subjectiviser la scène est une montée en puissance des gongs, comme un bruit assourdissant dans le cerveau du personnage.

Nous le voyons: les effets de son, de voix et de caméra instaurent un masque, une dissymétrie entre les deux sens auditif et visuel, des dissymétries pour dire les maux de l'âme et les troubles du corps. Michel Chion soulignait le rôle des perceptions transsensorielles dans le son ${ }^{28}$ et cet aspect est déterminant dans tous les films cités où les sens sont mis à mal, parfois jusqu'à la léthargie.

Premier indice de l'importance des sens, en référence à Mapa de los sonidos de Tokio, Isabel Coixet avoue: "Je crois que le sexe et la nourriture sont très liés dans le film ${ }^{29}$ ". Toutefois, dans une certaine mesure, Ryu est d'abord masculinisée, absente de son corps de femme, comme nous l'avons vu. La voix-off entraine le spectateur dans la pénombre d'un entrepôt (la Halle aux marées) où s'agitent des formes difficiles à identifier. La caméra suit finalement une jeune femme qui découpe, scie et prépare du poisson congelé. 


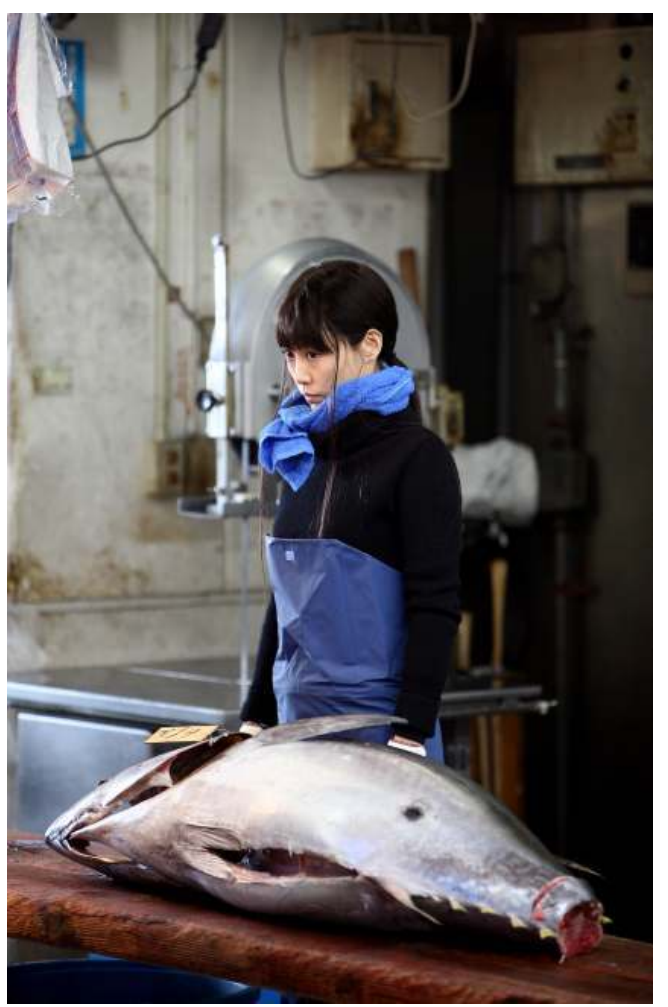

Figure 4

(c) 2009. MEDIAPRODUCCIÓN S.L.U. I VERSÁTIL CINEMA S.L.Fotógrafo : David Coll Blanco

Ensuite, la continuité du regard indiscret de la caméra et de la voix-off nous installe dans le quotidien du personnage. Mais ces scènes ont surtout l'intérêt de souligner le surinvestissement du regard auquel invite la voix-off et son lien au voyeurisme. Engagée pour tuer David, interprété par Sergi López, Ryu se retrouve dans l'incapacité de l'exécuter car cet homme l'éveille aux sens. Spécialiste de vin, il l'initie à la dégustation de ce breuvage, et c'est un choc pour elle. Une fois engagée dans cette dynamique kinesthésique, Ryu découvre le langage érotique de la nourriture et de la boisson. Pour Gilbert Duran, le vin « est symbole de la vie cachée, de la jeunesse triomphante et secrète. Il est par là, et sa rouge couleur, une réhabilitation technologique du sang. Le sang recréé par le pressoir est le signe d'une immense victoire sur la fuite anémique du temps ${ }^{30}$ ». L'élément liquide prend ici une nouvelle forme vitale et érotique: au vin dont les personnages se délectent s'ajoute le jus de citron dont s'imprègnent volontiers les corps féminins dans le film. Au contraire, la fille de Monsieur Nagara, Midori, utilise son propre sang pour accuser David d'être la cause de son suicide. Le sang de David doit être le prix de la vengeance.

Alors que les sens sont l'essence même de la constitution de soi dans Mapa de los sonidos de Tokio, l'absence précisément de stimuli sensoriels, dans La vida secreta de las palabras, invite à percevoir là une mise en péril de l'individuation. Certes, il s'agit de rendre le sensible dans ce parti pris de mettre en scène des êtres qui souffrent d'une infirmité : Hanna a perdu l'ouïe, Josef est atteint d'une cécité temporaire. Mais au-delà, l'incapacité d'Hanna à communiquer et à côtoyer les autres est une contrainte et, à la fois, un investissement de soi limité. En effet, elle apparaît, dans un premier temps, comme un 
personnage immuable, silencieux et enfermé dans une existence étriquée voire stérile dans ce qui est plutôt une négation du mouvement pour soi. La déficience auditive est ainsi le point de départ d'une organisation du récit autour de troubles sensoriels chez Hanna que les premières séquences mettent en exergue.

De ce point de vue, on constate une sorte d'agueusie chez le personnage principal, c'est-àdire un endormissement des cellules gustatives, quand le menu d'Hanna est invariablement le même : du riz, du poulet et une demi-pomme. Les premiers plans de ces aliments dans le réfectoire ou dans son frigidaire n'ont d'autre fonction que de marquer l'absence de variété alimentaire. Toutefois, le cuisinier de la plate-forme, qui cherche des mélanges savoureux, lui présente des épices, lui fait découvrir des mets et des saveurs insoupçonnées. Et brusquement, une fois seule, Hanna se surprend à dévorer littéralement le repas qu'a dédaigné Josef. Et la caméra de la filmer au rythme des vagues comme si tant de saveurs lui donnaient le tournis. La manière de la filmer est alors en concordance avec cette soudaine jouissance du palais, sensations kinesthésiques et équilibre du corps étroitement liés. À ce titre, Didier Anzieu a décrit et défini l'extrême importance de la peau et du corps dans la constitution et la préservation du Moi : «La peau, système de plusieurs organes de sens (toucher, pression, douleur, chaleur...) est elle-même en étroite connexion avec les autres sens externes (ouïe, vue, odorat, goût) et avec les sensibilités kinesthésiques et d'équilibration ${ }^{31}$ ».

Tout aussi naturellement, en découle, comme dans Mapa de los sonidos de Tokio, une synesthésie identifiable entre gustatif / odorat et odorat / toucher. Hanna ne se sépare jamais d'une douzaine de savonnettes: ce trouble obsessionnel compulsif tient de la phobie de la contamination et de la souillure. Mais se laver est aussi de l'ordre du toucher (soi et les autres) et de l'ordre de l'odorat. Puisque le savon permet également d'anesthésier toutes les odeurs étrangères, cela en appelle aussi à la perte de l'odorat, maladie dite de l'anosmie.

Sans doute faudrait-il se pencher par ailleurs sur cette image d'Hanna, toujours dans les premières séquences, qui téléphone à Inge sans lui adresser la parole. De suite, Inge identifie son interlocutrice silencieuse. Bien que l'on apprenne plus tard qu'Hanna s'est confiée à Inge et que ce témoignage a été enregistré, il y a une sorte d'aphasie volontaire dans la mesure où Hanna refuse de parler à cette femme dont le spectateur ne sait rien. Inge évoque alors les lettres qu'elle a écrites et qu'Hanna conserve (elle les apporte avec elle sur la plate-forme) tout en refusant de les décacheter. On pourrait voir dans ce refus du langage écrit, une incapacité à le comprendre, maladie que l'on nomme alexie.

En somme, tout se passe comme si Hanna cumulait des pertes partielles ou complètes de fonctions vitales, toutes liées à la communication. Elle est d'emblée placée sous le signe du manque : manque d'ouie, de communication, de compagnie et d'envies.

52 À partir de là, de nombreuses relations se tissent entre ce qu'elle fait et ce qu'elle ne ressent plus. Elle accepte de prendre soin de Josef, victime de brûlures graves dans l'incendie filmé en ouverture du récit. Hanna soigne, lave, alimente, écoute et donne la parole à cet homme diminué. Force est de constater l'implication essentielle de la peau et de l'organe buccal dans leurs rencontres. En effet, pendant les soins, Josef se confie à l'étrangère et déverse un flot de paroles visant à le libérer de sa culpabilité d'être en vie. Mais dans ce jeu de confessions de l'autre et de soi, c'est le récit de la souffrance silencieuse d'Hanna qui se construit en filigrane. Hanna retrouve les sens seulement après avoir trouvé, après avoir sauvé l'autre. 

voué à la solitude et au repli, où elle perd son inhibition. Le contact se crée au fur et à mesure qu'elle est celle qui prodigue des soins et celle qui porte son attention à chaque habitant de la plate-forme. Se succèdent donc des scènes dans lesquelles Hanna écoute Dimitri (le capitaine) lui faire le récit de l'accident, questionne Martin (l'océanographe) sur ses mesures, ou regarde les photos de famille que lui montre Liam. Qui plus est, une réelle complicité se crée entre elle et Simón (le cuisinier) comme lorsqu'ils s'interpellent avec tendresse et humour, oscillant au même rythme sur une balançoire. Cela signale aussi la métamorphose d'Hanna, capable enfin de rire.

Si toutes ces scènes alternent avec des plans d'ensemble de la plate-forme depuis la mer, c'est pour mieux exprimer le temps qui passe et combien le personnage est émotionnellement ébranlé. Dans ces circonstances, rien d'étonnant à l'omission par la cinéaste des interventions de la voix-off incluses dans le scénario, car l'importance est donnée à l'échange entre Hanna, si souvent filmée en plans rapprochés et en gros plans, et les autres individus.

\section{La voix-off et la témérité de l'indicible}

Est-ce la trame filmique qui crée la nécessité de la voix-off, ou celle-ci parvient-elle à transcender le récit? Autrement dit, que gagne le récit à s'enchâsser dans une voix-off?

56 À cet égard, il nous parait éclairant de reconsidérer le paradigme développé par Paul Ricœur, dans Temps et récit, paradigme des trois Mimèsis, en tant que mimèsis d'action. Tout d'abord, la forme narrative, selon Ricœur, s'enracine dans une pré-compréhension du monde de l'action. Il s'agit de Mimèsis I, qu'il développe dans le Tome I (les conditions du récit). Le deuxième niveau, Mimèsis II, est celui de la configuration du récit. C'est ce que Ricœur désigne par le terme de « mise en intrigue », soit un agencement des faits avec un début, un déroulement et une fin de manière à ce qu'ils prennent un sens, qu'ils soient «capables d'assurer la pérennité de raconter ${ }^{32}$ ». Chez Isabel Coixet, la voix-off, systématiquement en ouverture et en épilogue du récit, lui adjoint une apparente structure circulaire. Enfin, Mimèsis III est la re-figuration du passé, entendue comme la séquence par définition inachevée, et ouvrant des réinterprétations possibles. Cette conception du narratif met l'accent sur deux points fondamentaux: la fonction de l'action et celle du sens.

57 Ces deux fonctions sont systématiquement mises en jeu dans les films d'Isabel Coixet : la voix-off y exerce cette fonction herméneutique en donnant du sens à l'insensé, en transformant une situation de passivité en action. «Tout point de vue est l'invitation à un lecteur à diriger son regard dans le même sens que l'auteur ou le personnage ; en retour, la voix narrative est la parole muette qui présente le monde du texte au lecteur ${ }^{33}$ " soulignait Paul Ricœur. Les récits d'Isabel Coixet sont bel et bien structurés selon une ligne narrative définie et un enchaînement linéaire auxquels participe la voix narrative. En conséquence, entre mémoire et prise de conscience, la voix-off enclenche un effet « thérapeutique » du récit dans une configuration pluridimensionnelle éclairante. Elle fait le lien entre l'histoire vécue et l'histoire tue, elle dit les mots du silence. Ne serait-ce pas là le sens du titre, La vida secreta de las palabras?

De façon récurrente encore, dans l'œuvre d'Isabel Coixet, le personnage se présente au spectateur selon un mouvement brisé. Comme nous l'avons vu précédemment, la voix-off

Cahiers de Narratologie, 22 | 2012 
entre d'emblée en jeu combinée à des fondus au noir et au ralenti. Le premier niveau permet ainsi de montrer un sujet amputé d'une partie de lui-même, fragilisé dans son entité humaine. Un deuxième niveau serait son incapacité initiale à s'auto-ériger moteur de son propre destin d'où cette répétition du personnage, en miroir, qui s'exhibe de dos, de profil, mais toujours partiellement. La voix-off soutient elle-même ce paradigme de l'être isolé de lui-même puisqu'elle est la voix détachée d'un corps inaccessible. Mais elle annonce tout autant la révolte finale contre la menace de l'anéantissement de soi. À la fin de la première séquence de Mi vida sin mí, la voix-off féminine déclare : «Ésta eres tú »; il s'agit d'ÊTRE véritablement et sans concession.

59 L'examen attentif des premières séquences de Cosas que nunca te dije, Mi vida sin mí, La vida secreta de las palabras et Mapa de los sonidos de Tokio, forment un ensemble cohérent depuis ce point de vue. Les fondus au noir découpent les plans de personnages, mais la réalisatrice joue également sur une gamme chromatique réduite. Ainsi, les premières images de Cosas que nunca te dije parcourent, depuis un drap blanc abandonné, le corps d'un homme enveloppé dans une chemise blanche, tel un linceul, et recroquevillé presque dans une position foetale. Comme à l'accoutumé, le travelling en plongée totale (depuis le plafond) qui avance le long de ce corps inerte est entrecoupé par les fondus au noir, et par le plan d'une tasse de café noir qui éclabousse des pages blanches au ralenti. Toutefois, le mouvement est marqué par le frémissement d'un lac au coucher du soleil que contemple un homme de dos. Autant de signes de perte de soi, comme nous l'avons dit. Le procédé se répète dans Mi vida sin mí à un détail près : le premier impact de la voix-off évoque ici des yeux fermés : « Ésta eres tú, los ojos cerrados », mais la première image est une ouverture au blanc. La caméra détaille ensuite une femme debout sous la pluie à qui associer la voixoff.

\section{Mi vida sin mí}

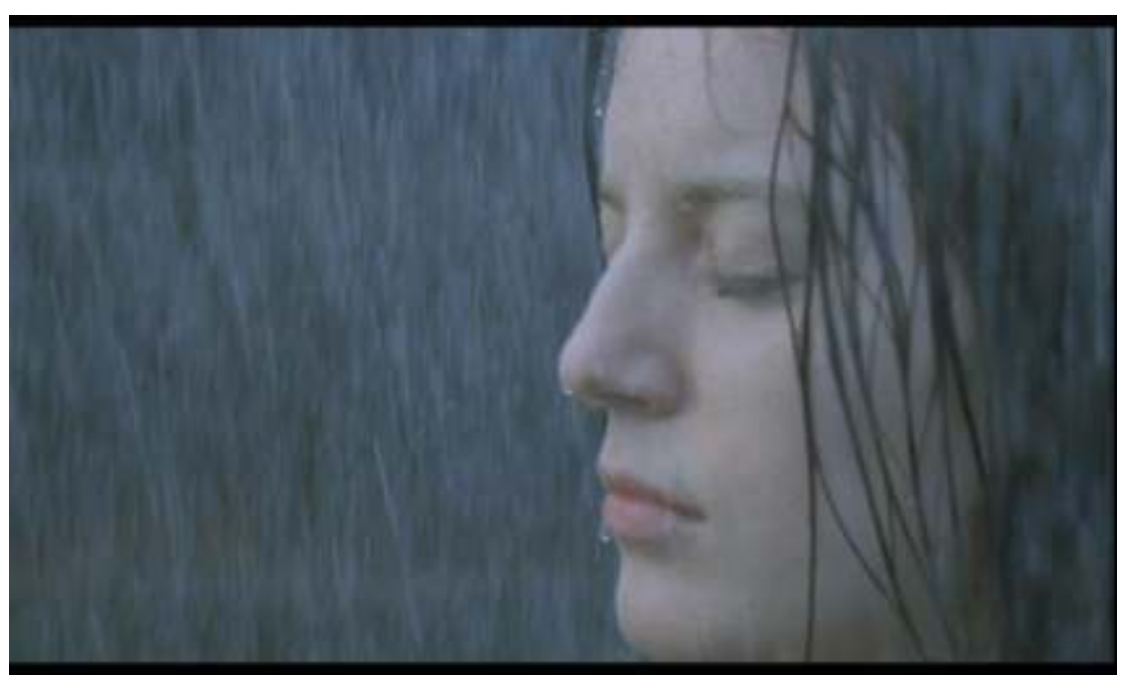

Figure 5

(C) EL DESEO, D.A., S.L.U

La caméra l'évalue, la jauge sans qu'elle ne nous adresse le moindre regard. C'est que la voix-off chez Isabel Coixet est incompatible avec le regard-caméra trop subjectivant ou peut-être trop peu original pour elle. Elle aspire à un niveau supérieur du visuel dans un façonnage à peine perceptible de la perception du spectateur. Le dispositif associé à la voix-off renvoie à ces «images sonores» que Laurent Jullier définit comme «des 
représentations psychologiques d'entités sonores présentant un comportement acoustique cohérent ${ }^{34}$ ». Ce dispositif trouve son aboutissement dans La vida secreta de las palabras où elle acquiert une puissance que nous qualifierons de téméraire. Action et sens se mêlent ici immanquablement car il s'agit de raconter l'irracontable : la torture et la mort.

61 Et soudain l'aphasie, le mutisme d'Hanna se transforme en longue confession qu'écoute un Josef abasourdi. Le schéma narratif linéaire de leur rencontre est brutalement brisé par le récit-confession d'une victime de la guerre des Balkans. Pourtant, ce récit, reconstitué et re-constituant, refuse encore sa place à Hanna car elle exclut l'association de ces horreurs à sa propre personne. Elle raconte alors les sévices infligés à son amie, la tristesse et la mort d'une femme obligée de tuer son enfant, les hurlements des autres femmes...

Enfin, quand Josef, bouleversé, lui demande le nom de son amie, elle la nomme par son propre prénom, « Hanna ».

63 Paradoxalement encore, c'est celui qui est aveugle, Josef, qui va toucher sur le corps d'Hanna ce que la parole ne peut plus dire. Le signe corporel (les cicatrices sur la poitrine d'Hanna) figure le traumatisme dont le souvenir avait été jusque là refoulé. Néanmoins, Hanna continue d'identifier l'amie comme victime des entailles que Josef parcourt de ses mains. Elle rejette hors d'elle le pronom sujet « je » trop intime et transcrit la perte de celle qui parle par l'utilisation du «tu » : «Sais-tu ce que c'est? Qu'on te viole une fois et encore une fois ${ }^{35}$ ? », ce qui traduit parfaitement la perte de soi. Il s'agit là d'un processus commun aux victimes de torture car le tortionnaire ébranle l'identité même de l'être humain ${ }^{36}$. C'est l'anéantissement de la personne par des attaques répétées du corps et du sentiment d'humanité, par la négation des besoins, des liens - et Hanna spécifie que ses tortionnaires appartenaient à l'armée de son pays, parlaient la même langue qu'elle.

Selon la Déclaration contre la torture adoptée par les Nations Unies le 9 décembre 1975, elle « désigne tout acte par lequel une douleur ou des souffrances aiguës, physiques ou mentales, sont délibérément infligées à une personne ${ }^{37}$ ». Le sentiment d'impuissance est total dès lors qu'il n'y a plus d'aide à attendre de quiconque.

Mais la notion de culpabilité sous-tend la narration d'Hanna. La solidarité a été blessée du fait que la survivance engendre moralement la culpabilité ; si je survis alors que l'autre est tué, je suis coupable. Le raconter, se raconter est encore une manière de repousser, d'éloigner le souvenir de ce qui est arrivé. Manœuvre peut-être encore vaine, car comme le dit Inge, Hanna fait partie de « ceux qui ont honte d'avoir survécu (...) et cette honte, qui est plus grande que la douleur, plus grande que tout, peut demeurer à tout jamais ${ }^{38}$ ". Au contraire, la confession d'Hanna remplit la fonction essentielle qu'attribue Françoise Sironi à la thérapie des victimes de la torture: trouver un interlocuteur. Et un interlocuteur agissant: Josef lui promet qu'il apprendra à nager pour la sauver de ses propres larmes. Et ce « aprenderé » libère l'accès à la dimension humaine par excellence ; l'avenir. Par sa promesse, Josef lui transmet l'instinct non plus de survie mais l'instinct de vie, tout simplement.

Quelle est alors l'implication de la voix-off dans cette douloureuse confession?

Pour mieux saisir l'articulation de la voix-off et du récit, il faut rappeler que la voix d'enfant, apparue dans la première séquence, disparaît au moment précis où atterrit, sur la plate-forme pétrolière, l'hélicoptère qui dépose Hanna, engagée comme infirmière. Elle réapparaît dans la dernière séquence et acquiert une dimension particulière qui est de 
l'ordre de la résonance. Tandis que la caméra filme, au ralenti, deux enfants qui courent vers nous à travers une vitre segmentée, la voix-off, une dernière fois, supplée aux défaillances du récit.

La vida secreta de las palabras

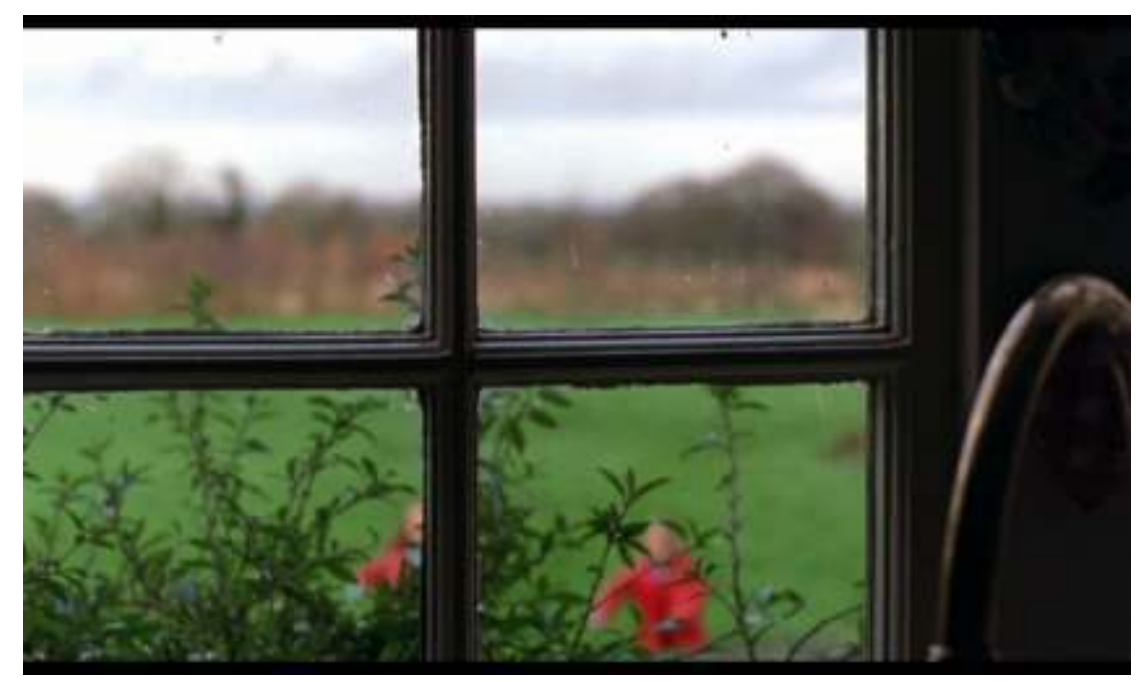

Figure 6

(c) EL DESEO, D.A., S.L.U

68 À nouveau, l'apparence de la réalité est donnée à la voix-off, proche et claire, à la différence des enfants dont les rires nous parviennent comme lointains et dont l'image nous parvient à travers le verre dépoli de la vitre. A mesure que les enfants s'approchent au ralenti, la voix-off dit s'éloigner, «Je m'éloigne, je suis déjà loin. Peut-être que je ne reviendrai jamais ${ }^{39} »$. Il y a là confusion des espaces et des perceptions.

À vrai dire, la voix-off ne prend tout son sens qu'une fois le silence brisé, qu'une fois le film achevé, lorsqu'elle identifie les enfants comme ses frères : « Elle a maintenant deux enfants, mes frères ${ }^{40} »$. Elle acquière sa dimension signifiante à partir de l'adjectif possessif. D'une certaine manière, le spectateur reconstruit les pièces manquantes du puzzle et se laisse mener au-delà des images « Je reviens alors vers elle. Elle me berce, elle me caresse les cheveux. Et rien, rien de tout ce qui s'est passé ne s'interposera plus entre nous ${ }^{41}$ ». Et si Hanna était en réalité cette femme poussée par ses tortionnaires à tuer sa fille? Tout repose de la sorte sur une stratégie remarquable du film quand l'énonciation (que Ricœur désigne comme le discours du narrateur), celle de la voix-off, reformule l'énoncé (celui d'Hanna). Cela permet de prendre toute la mesure de la parole dévoilée et de la parole refoulée, toutes deux conduites par une voix acousmatique qui dit les mots du silence et dramatise le silence. Et cette voix-là ne peut s'incarner.

Dans le creux de l'absence, il y a une présence. Disons qu'au-delà de la fixation au traumatisme, la voix-off révèle l'incorporation d'un objet perdu à la réalité : «Elle n'a jamais vu mon visage. Mais je suis sa seule compagnie. Elle me voit vêtue d'une salopette en velours rouge, d'un polo en coton bleu clair. La salopette est toujours rouge. Et le polo, toujours bleu. Pourquoi? Elle ne sait pas pourquoi ${ }^{42}$ ». On peut lire chez Freud que "l'épreuve de la réalité a montré que l'objet aimé n'existe plus et elle somme alors l'endeuillé de soustraire toute sa libido de ses attachements à cet objet ». La résistance naturelle est alors de s'accrocher à l'objet «par une psychose hallucinatoire de désir ${ }^{43}$ ". 
Ainsi, simultanément au désinvestissement de soi, il y a réinvestissement de l'être aimé et perdu, d'où cette limpidité, cette réalité accordées à la voix-off. Désormais, Hanna est la seule capable d'entendre la voix de l'enfant et de la désacousmatiser, si l'on peut dire. En même temps, la réactualisation, voire l'identification, agissent comme mirages de la continuité de soi, d'où cette accumulation de signes de la perte de soi.

Quand Freud distingue le deuil de la mélancolie, il signale que les deux états ont en commun une humeur profondément douloureuse, un désintérêt pour le monde extérieur, la perte de la faculté d'amour, l'inhibition. S'ensuit l'abandon de toute activité et de tout intérêt pour ce qui est extérieur. À ceci s'ajoute dans la mélancolie le refus de s'alimenter, et un dernier trait distinctif réside dans sa tendance maniaque. Comment ne pas voir à cet égard, dans le personnage d'Hanna, une cristallisation douloureuse de ces symptômes qui vide le Moi jusqu'à son appauvrissement?

Au-delà de ce que Paul Ricœur nomme «ce pouvoir de rouvrir le passé sur l'avenir ${ }^{44}$ », la voix-off complète la confession salvatrice et révèle le sens du vécu, par exemple ce qui a été tu dans la confession du personnage dans La vida secreta de las palabras. Une confession qui lui permet malgré tout de redevenir sujet et non plus seulement objet-victime.

Chacun des films, à sa manière, manifeste l'artifice de la voix-off qui s'exprime quand l'agir est impossible et quand le silence est une limite. À notre sens, le recours systématique à la voix-off agit comme une expérimentation de mise en relations possibles et élabore un récit ouvert pour lui donner du sens et le rapprocher de la sensibilité du spectateur. Il n'en est pas moins vrai que le rôle de la voix-off chez Isabel Coixet est de témoigner, non pas seulement de l'émotion, mais de surdimensionner des forces émotionnelles insoupçonnées. Elle permet aussi de déterminer les stratégies que l'auteur peut mettre en place pour s'accommoder des contraintes, pour les contourner, voire pour en faire un moteur du processus créatif.

Nous citerons à cet égard une dernière fois Michel Chion : «Ces sons et ces voix qui ne sont ni tout à fait dedans, ni clairement dehors, on comprendra vite que ce sont eux qui nous intéressent le plus : parce que c'est là peut-être, avec ces voix et ces sons laissés en errance à la surface de l'écran, qu'entre en jeu la puissance du cinéma en tant que tel ${ }^{45}$ ».

\section{NOTES}

1. Michel Chion, La voix au cinéma, Paris, Éditions de l'Étoile/Cahiers du Cinéma, coll. (Essais), 1993, p. 18.

2. A los que aman (À l'heure des nuages): Au XVIIIème siècle, un vieillard raconte à un jeune homme l'histoire d'une grande passion : celle qu'il a éprouvée dès l'enfance pour Matilde qui est morte d'amour et qu'il n'a pas su guérir.

3. Jean-Louis Alibert, Le Son de l'image, Grenoble, PUG, 2008, p. 83.

4. Michel Chion, Le Son, Paris, Armand Colin, 2006, p. 11.

5. Michel Chion, Le Son, op. cit., p. 32.

6. «Pasé mi vida amando a una mujer que amaba a otro que no la amaba a ella sino a otra de la que nunca supe si le correspondía ». (Traduction de l'auteur). 
7. Il dépose des bouquets de fleurs et des billets doux anonymes à destination de Matilde jusqu'à ce que celle-ci identifie, par erreur, son admirateur sous les traits de Léon.

8. «Dicen que a través de las palabras el dolor se hace más tangible. Yo siempre he creído que el dolor que no encuentra palabras para ser expresado es el más cruel, el más hondo, el más injusto ». (T.d.a.).

9. Mapa de los sonidos de Tokio (Carte des sons de Tokyo) : Ryu est une fille solitaire dont l'air fragile contraste avec la double vie qu'elle mène. La nuit, elle travaille dans la Halle à Marée de Tokyo mais elle est aussi occasionnellement tueuse à gages. Mr Nagara est un entrepreneur puissant, affligé par la mort de sa fille Midori qui s'est suicidée. Il en rend responsable David, un homme d'origine espagnole qui tient un commerce de vins à Tokyo. Ishida, un employé de Mr Nagara, qui aimait en secret Midori, engage Ryu pour tuer David.

10. Gérard Genette, Nouveau discours du récit, Paris, Éditions du Seuil, 1983, p. 49.

11. «Eso creo recordar ", « por supuesto, estoy casi seguro ». (T.d.a.).

12. " $i$ Os lo he dicho antes?». (T.d.a.).

13. En réalité, il s'agit du marché aux poissons d'Oda qui a donné toutes sortes de facilités de tournage à l'équipe d'Isabel coixet.

14. «Cuando la conocí se llamaba Ryu. [...] Nos conocimos un domingo por la tarde en el Museo del Ramen ». (T.d.a.).

15. « $¿$ Me dejas grabar el ruido que haces ? En realidad me dedico a eso. Así me gano la vida.

- ¿Te ganas la vida así ?» (T.d.a.).

16. Jean-Louis Alibert, op. cit., p. 77.

17. « No hay día en que no recuerde la respiración de Ryu ni días que eche de menos su silencio», «Comíamos juntos siempre en silencio ». (T.d.a.).

18. «Alguien dijo que desde el momento en que uno tiene vida interior ya está llevando doble vida ». (T.d.a.).

19. Michel Chion, Le Son, op. cit., p. 10.

20. Ibid., p. 30.

21. N'apparaissent que les maisons de production, les producteurs et la réalisatrice. Un générique complémentaire défilera après la séquence que nous allons analyser.

22. La chanson Hope There's Someone, titre évocateur pour notre propos, est interprétée par Antony \& The Johnsons.

23. «Es una voz de gran pureza, que habla desde algún punto más allá del "aquí y ahora" que dejan ver las imágenes ", Isabel Coixet, La vida secreta de las palabras, guión, Barcelone, Ediciones B, 2005, p. 13. (T.d.a.).

24. «En el fondo hay tan pocas cosas. Millones y millones de agua. Rocas y gas. Afecto. Sangre. Cien minutos. Mil años. Cenizas. Luz. Ahora. Ahora mismo. Hace un momento... ¿ Os lo he dicho antes? ¿ Hay muy pocas cosas. Silencio. Y palabras. »

25. La version espagnole est plus ambiguë : «En el fondo ». Au fond des choses ou au fond de la mer?

26. Les informations générales du générique apparaissent en surimpression au fil d'une promenade nocturne dans la baie de Tokyo.

27. "¿ Por qué lo ha hecho?». (T.d.a.).

28. "Qui ne sont d'aucun sens en particulier, mais peuvent emprunter le canal d'un sens ou d'un autre, sans que leur contenu et leur effet soient enfermés dans les limites de ce sens ». Ibid., p. 56.

29. Interview réalisée par Alejandro Ciriza, 28.08.2009, El País, « Yo creo que el sexo y la comida van muy unidos en la película ». (T.d.a.).

30. Gilbert Duran, Les structures anthropologiques de l'imaginaire, Paris, Dunod, 1992, p. 298.

31. Didier Anzieu, Le Moi-peau, Paris, Dunod, 1995, p. 36.

32. Paul Ricœur, Temps et Récit, Paris, Éditions du Seuil, 1984, Tome II, p. 294.

33. Ibid, p. 188. 
34. Laurent Jullier, Cinéma et cognition, Paris, L'Harmattan, 2002, p. 52.

35. « ¿ Sabes lo que es ? Qué te violen, una y otra vez ». (T.d.a.).

36. Cf. Françoise Sironi, dans sa pratique de psychothérapeute et d'ethnopsychiatre, s'est spécialisée dans le soin des victimes de tortures. Cf. Bourreaux et victimes, Paris, Odile Jacob, 1999, pp. 63-77.

37. Cf. Amnesty international, La Torture, instrument de pouvoir, fléau à combattre, EFAI, Éditions du Seuil, 1984, p. 26.

38. «Los que se avergüienzan de haber sobrevivido (...) y esa vergüenza, que es más grande que el dolor, que es más grande que cualquiera cosa, puede durar para siempre ». (T.d.a.).

39. «Me alejo, ya estoy lejos. Quizás no vuelva nunca ». (T.d.a.).

40. «Tiene dos niños ahora, mis hermanos». (T.d.a.).

41. «Entonces, vuelvo a ella. Me acuna y me acaricia el pelo y ya nada, nada de lo que ocurrió volverá a interponerse entre nosotros. » (T.d.a.).

42. «Nunca ha visto mi rostro. Me ve con un peto de pana rojo y un jersey de cuello alto claro. El peto siempre es rojo y el jersey siempre azul. ¿ Por qué ? Ella no sabe por qué. » (T.d.a.).

43. Sigmund Freud, Deuil et mélancolie, Paris, Éditions Payot, 2011, p. 47.

44. Paul Ricœur, La mémoire, l'histoire, l'oubli, Paris, Éditions du Seuil, 2000, p. 495.

45. Michel Chion, La voix au cinéma, op. cit., p. 18.

\section{RÉSUMÉS}

Le recours presque systématique de la voix-off chez Isabel Coixet laisse présupposer une unité de sens qui reste à démontrer. L'originalité de A los que aman (1997), La vida secreta de las palabras (2005) et El mapa de los sonidos de Tokio (2009)apparaît dans leur capacité à montrer une voix qui semble incarner la présence mais renvoie paradoxalement à l'absence. De fait, on pourra s'interroger sur l'acte de parole comme véritable lien entre le corps et le code quand la voix-off complète une confession salvatrice et révèle le sens du vécu. En effet, entre mémoire et prise de conscience, la voix-off dit surtout la douleur de la situation traumatique. Mais est-elle pour autant l'instrument de la catharsis?

El empleo casi sistemático de la voz en off en la obra de Isabel Coixet ofrece una unidad de sentido que incita a buscar más allá. La originalidad de tres películas en concreto - A los que aman (1997), La vida secreta de las palabras (2005) et El mapa de los sonidos de Tokio (2009)- emerge de su capacidad a mostrar una voz que parece remitir a una presencia y sin embargo remite paradójicamente a una ausencia. De hecho, cabe interrogarnos sobre este acto de palabra que conlleva una verdadera unión del cuerpo y del código cuando la voz en off completa una confesión que ha de salvar y revela pues el sentido de lo vivido. En efecto entre memoria y toma de conciencia, la voz en off confiesa sobre todo el dolor del trauma. ¿ Apunta entonces tal proceso a la catarsis inadvertida en la confesión? 
INDEX

Index géographique : Espagne

Mots-clés : absence, confession, deuil, silence, traumatisme, voix-off

Index chronologique : 1997-2009

\section{AUTEUR}

\section{SILVINA BÉNÉVENT GONZÁLEZ}

Silvina Bénévent González est Docteur en Études Culturelles de l'Université Paul ValéryMontpellier 3. Maître de conférences, elle appartient au groupe LCE-CETIAL de l'Université Lumière Lyon II. Ses domaines de recherche sont le cinéma, la littérature contemporaine et la psychanalyse. Elle s'est notamment intéressée à l'œuvre de Carlos Saura et de Claudia LLosa en cinéma et celle de Juan Marsé et de Carlos Liscano en littérature. 